\title{
Intonation and polar questions in Greek
}

Anthi Chaida, Angeliki Sotiriou, Athina Kontostavlaki

Laboratory of Phonetics and Experimental Linguistics, University of Athens, Greece

https://doi.org/10.36505/ExLing-2016/07/0009/000268

\begin{abstract}
The present study focuses upon the effects of lexical stress and focus on Greek polar (yes/no) questions. According to the results of a production experiment, the tonal structure of neutral questions presents striking similarities with the tonal structure of questions with focus on the final element. Questions with focus in the first element display a different tonal structure and do not show the typical F0 fall on the stressed syllable of the nucleus. The peak of the tonal boundary in these questions aligns with the last stressed syllable, while in neutral questions and in questions with focus in the final element it aligns with the last syllable of the utterance.

Key-words: intonation, polar questions, lexical stress, focus, Greek.
\end{abstract}

\section{Introduction}

This study aims to investigate the interaction of lexical stress and focus with the intonation of polar (yes/no) questions in Greek. Although different sentence types and specifically polar questions as well as focus have been the objective of several studies (e.g. Chaida 2010), the effect of the position of lexical stress on tonal contours, and especially on tonal boundaries, still remains an open question (see Botinis et al. 2016). According to previous studies, the tonal structure of polar questions consists of a low nuclear tone, followed by a risin-falling tonal movement at the right edge of utterances. More specifically, the tonal peak has been found to align either with the last syllable of the sentence when focus in the last word or with the last stressed syllable when focus earlier (Grice et al., 2000, Arvaniti 2002, Baltazani 2007, Chaida 2010).

\section{Experimental methodology}

One simple sentence was crossed with 3 focus renditions (no focus, focus on the first element, focus on the final element), and 3 lexical stress placements on the final element (Table 1). The speech material was placed in 3 lists with random order, and was produced by 10 female speakers aged 20-40 years old with standard Athenian pronunciation. The speakers were given verbal instructions and provided with contextual information and a suggested answer for every question. The total corpus of the recorded utterances consisted of 270 utterances (3 sentences X 3 focus renditions X 10 speakers X 3 repetitions).

ExLing 2016: Proceedings of $7^{\text {th }}$ Tutorial and Research Workshop on Experimental Linguistics, 27 June - 2 July 2016, Saint Petersburg, Russia 
The speech productions were directly recorded into a computer hard disk at the isolated sound recording booth of the Laboratory of Phonetics and Computer Linguistics of the University of Athens. The speech material was analyzed with Praat software, and the relevant data were automatically generated through the script Prosody Pro (Version 5.6.0) (Xu 2013). MS Excel and a Python script were used for the creation of graphs.

Table 1. Speech material of polar questions used for recordings, based on 3 different lexical stress placements crossed with 3 focus renditions (in bold)

\begin{tabular}{|c|c|c|}
\hline STRESS-SYLLABLE & FOCUS & TARGET UT'TERANCE \\
\hline antepenultimate & No focus & 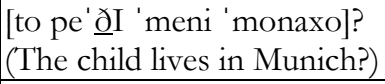 \\
\hline antepenultimate & First element & [to pe' $\underline{\partial i}$ 'meni 'monaxo]? \\
\hline antepenultimate & Final element & [to pe' $\underline{\text { i }}$ 'meni 'monaxo]? \\
\hline penultimate & No focus & $\begin{array}{l}{[\text { to pe' } \underline{\text { di 'meni mo' naxo]? }}} \\
\text { (The child lives alone?) }\end{array}$ \\
\hline penultimate & First element & 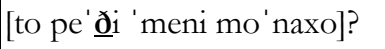 \\
\hline penultimate & Final element & [to pe' $\underline{\text { i }}$ 'meni mo'naxo]? \\
\hline ultimate & No focus & $\begin{array}{l}\text { [to pe' } \underline{\text { oi }} \text { 'meni mona'xo]? } \\
\text { (The child lives alone?) }\end{array}$ \\
\hline ultimate & First element & [to pe' $\underline{\partial i}$ 'meni mona'xo]? \\
\hline ultimate & Final element & 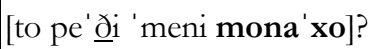 \\
\hline
\end{tabular}

\section{Results and discussion}

Figures 1-3 show the results of the present investigation. In accordance with these results, the position of lexical stress in the last word of the sentence and the position of focus affect the tonal structure of the question. In final focus and in neutral utterances, the position of lexical stress affects the alignment of the peak of the large F0 rise and consequent fall. The earlier the last lexical stress of the utterance, the earlier the aforementioned peak aligns within the utterance and the larger the inclination of the pitch curve.

As far as focus-first utterances are concerned, the position of the lexical stress of the final word is related with the position of the peak of the tonal boundary, which co-occurs with the final stressed syllable. In addition to this and contrary to the results of previous studies (e.g. Grice et al. 2000, Arvaniti 2002), instead of a fall of the value of the F0 on the stressed syllable of the word in focus, there is a rise in pitch. 
Furthermore, there appears to be no tonal range expansion associated with the word in focus, as the case is for declaratives(Botinis et al. 2001).

Neutral and final focus utterances display similar tonal structures, because the nucleus is aligned in both cases with the right prosodic edge and not with the verb. Consequently, there can be no post-focal deaccenting. Regarding the alignment of the nucleus in neutral questions, the results of the present study differ from the results of previous studies, where the nucleus is in the verb (Baltazani 2007, Chaida 2010).

As to the effect of focus on tonal boundaries, the peak of the tonal boundary in questions with nucleus on the final word is aligned with the last syllable of the utterance in all cases. On the other hand, in questions with an early nucleus, the peak of the tonal boundary is aligned with the last stressed syllable. This finding is in line with previous studies (Grice et al. 2000, Arvaniti 2002, Baltazani 2007, Chaida 2010).

Focus last and neutral questions were produced, in general, in a consistent way and focus was given as expected. On the contrary, in early focus questions, focus was either given in the first element, as required ( $\sim 62 \%$ of the utterances) or in both the beginning and the end of the utterance $(\sim 38 \%)$. Considering the above, further research on polar question prosodic features is required, since it seems that it still remains an open issue.
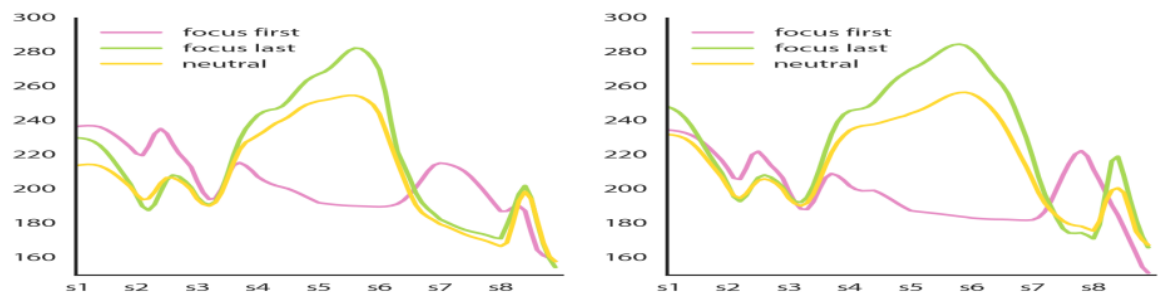

Figure 1a. Intonation of polar questions Figure 1b. Intonation of polar questions with stress on the antepenultimate syllable with stress on the penultimate syllable in 3 in 3 focus renditions. focus renditions.

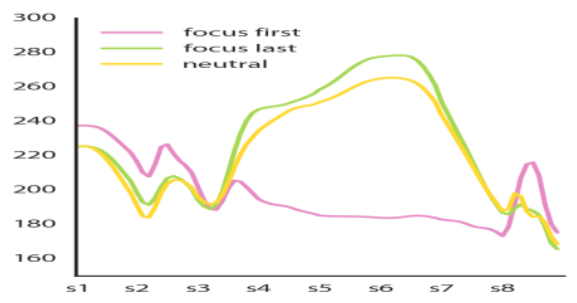

Figure 1c. Intonation of polar questions with stress on the ultimate syllable in 3 focus renditions. 

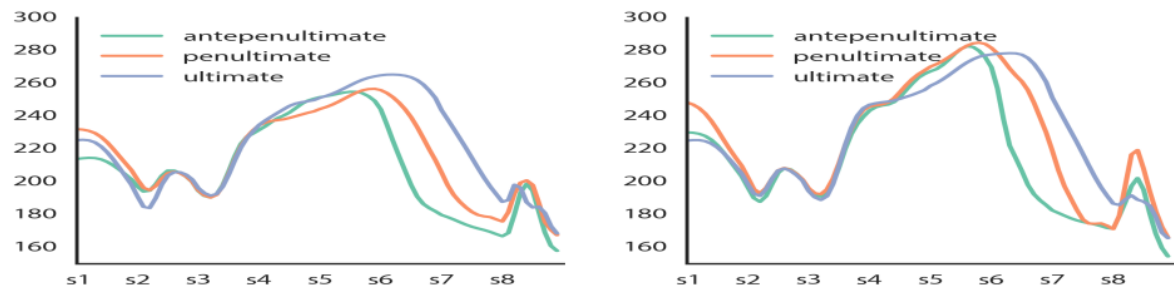

Figure 2a. Intonation of polar questions Figure 2b. Intonation of polar questions without focus, with 3 lexical stress with focus as well as 3 lexical stress placements on the final element.

placements on the final element.
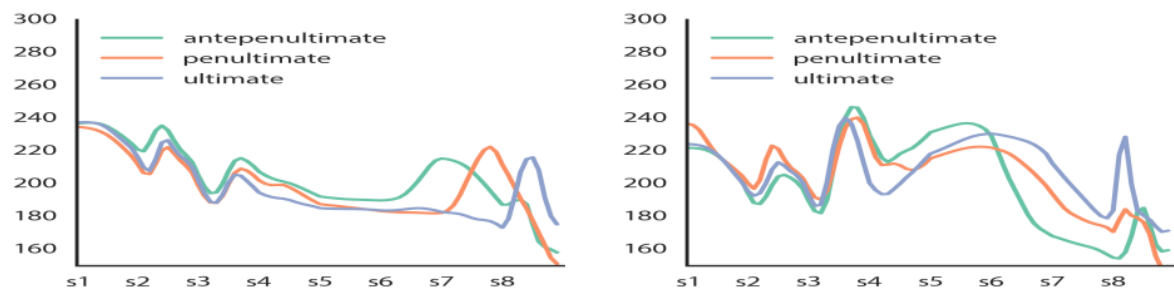

Figure 3a. Intonation of polar questions Figure 3b. Intonation of polar questions with focus on the first element and 3 lexical with focus on the first element and 3 stress placements on the final. (Realisation lexical stress placements on the final A, $62 \%$ of the utterances).

(Realisation B, 38\% of the utterances).

\section{References}

Arvaniti, A. 2002. The intonation of yes-no questions in Greek. In M. Makri-Tsilipakou (ed.), Selected papers on theoretical and applied linguistics (71-83). Thessaloniki: Aristotle University.

Baltazani, M. 2007. Intonation of polar questions and the location of nuclear stress in Greek. In Carlos Gussenhoven \& Tomas Riad (ed.), Tones and tunes, Volume II: Experimental Studies in Word and Sentence Prosody (387-405). Berlin: Mouton de Gruyter.

Botinis, A., Chaida, A., Nikolaenkova, O., Nirgianaki, E. 2016. Intonation and polar questions in Greek revisited. Proc. ExLing 2016 (this volume).

Botinis, A. Granström, B., Möbius, B. 2001. Developments and paradigms in intonation research. Speech Communication 33 (4), 263-296.

Chaida, A. 2010. Production and Perception of Intonation and Sentence Types in Greek. PhD Thesis, University of Athens.

Grice, M, Ladd, R. \& Arvaniti, A. 2000. On the place of phrase accents in intonational phonology. Phonology 17, 143-185.

Xu, Y. 2013. ProsodyPro - A Tool for Large-scale Systematic Prosody Analysis. In Proceedings of Tools and Resources for the Analysis of Speech Prosody (TRASP 2013), Aix-en-Provence, France.7-10. 